TOPICAL REVIEW

\title{
Surface induced ordering effects in soft condensed matter systems
}

\section{Peter Lang}

Forschungszentrum Jülich, Institut für Festkörperforschung, 52425 Jülich, Germany

\begin{abstract}
The symmetry break of the particle interaction field at surfaces causes a variety of interfacial effects including the variation of the relative stability of different phases of a given compound. In many materials surface melting is observed, i. e a surface near portion of the system is in a less ordered state than the bulk. In the last two decades, surface ordering, i.e. the stabilization of the higher ordered phase by the surface, has been observed for an increasing number of soft condensed matter systems. For melts of low molar mass compounds surface freezing was reported so far only for chain molecules, while in systems of colloidal length scale surface ordering has been observed for spherical particles as well. In this contribution we will review experimental studies, some theoretical approaches as well as computer simulations of surface ordering, both in the field of molecular melts and of colloidal suspensions.
\end{abstract}

E-mail: p.lang@fz-juelich.de 

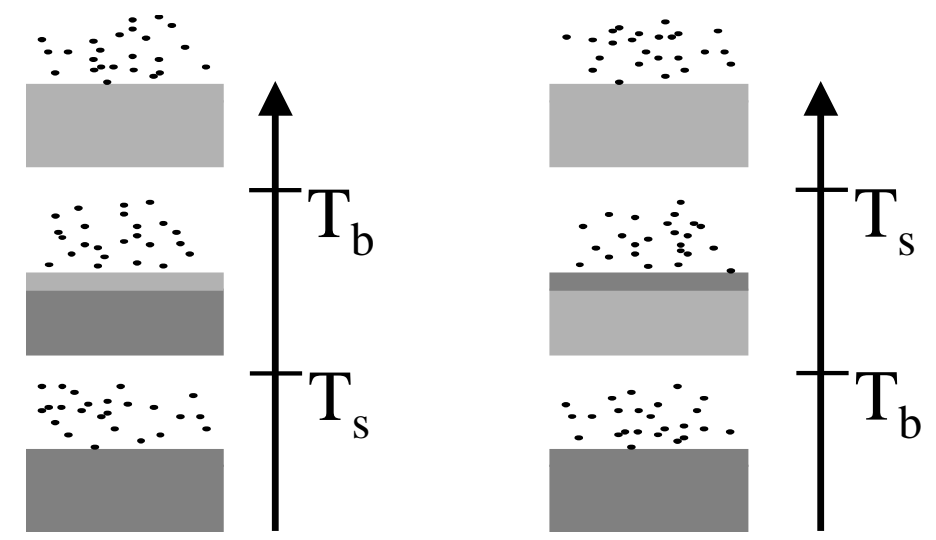

Figure 1. Schematic illustration of surface melting (left) where a less ordered (light grey) surface phase is in equilibrium with a more ordered (dark grey) bulk and the vapor phase at $T_{s}<T \leq T_{b}$; and surface ordering (right) where a more ordered surface phase is in equilibrium with a less ordered bulk and the vapor phase at $T_{b}<T \leq T_{s}$. The arrows indicate the direction of increasing temperature.

\section{Introduction}

The effect a surface or an interface may exert on the structure of an adjacent liquid phase has been subject of scientific interest for almost two centuries. It dates back to the thesis of Poisson of 1831 who first claimed that there must be a deviation of order parameters close to a surface from their bulk values. A historical review of this subject is given by Ninham [1]. With the advent of surface sensitive scattering techniques applying Lasers and synchrotron radiation it has become possible to study the structure of fluid interface with molecular resolution. Far away from thermodynamic conditions at which the bulk fluid undergoes disorder $\rightarrow$ order phase transitions (further called phase transition for simplicity), the structure of a liquid surface is mainly determined by a continuous density profile with an intrinsic thickness and thermally driven capillary waves. Experimental and theoretical contributions on this subject covering a large variety of systems from noble gases to metal melts were reviewed recently by Penfold [2].

If a liquid is in the vicinity of thermodynamic conditions (temperature, pressure and composition) at which phase transition occurs in the bulk, in a layer close to an interface the transition may be shifted to different values of the thermodynamic variables of state by an adjoining substrate or gas phase. Thus a surface phase is in equilibrium with a bulk phase and a spectator phase. The latter may be a substrate or a vapor phase. On approaching the thermodynamic conditions for the bulk phase transition the thickness of the surface layer may diverge into the bulk. In this case the surface phase is said to completely wet the spectator phase. If the surface phase wets the spectator phase incompletely it will maintain a constant thickness of only a few molecular length scales upon approaching the bulk transition.

In the vast majority of cases the surface phase is less ordered than the bulk phase as sketched in Figure 1. This situation is generally referred to as surface melting. It has been observed for a variety of metals [3-10] and molecular crystals. Especially the surface freezing of ice has been studied thoroughly [11-25]. 
The situation where a highly ordered surface phase is in equilibrium with a less ordered bulk and a spectator phase is called surface freezing or surface ordering. Up to now it appears that this phenomenon occurs only with anisometric molecules and consequently it was first observed with thermotropic liquid crystals. In one of the first systematic studies of the subject Miyano measured the surface induced enhancement of the nematic order parameter of an isotropic liquid crystal melt [26,27]. Later AlsNielsen et al demonstrated that a smectic film is formed on top of a nematic bulk of a liquid crystal between the surface transition temperature, $T_{s}$, and the bulk transition temperature, $T_{b}[28,29]$. In this case complete wetting of the vapor phase by the smectic film was observed. In the case of the isotropic/smectic A coexistence, Bahr and coworkers more recently found all types of known wetting behavior including first order wetting transitions $[30,31]$. Thermotropic liquid crystals are widely used in displays, the operating mode of which is based on interface induced ordering [32]. This is probably the reason why most systematic studies of surface ordering in soft condensed matter systems have been conducted on thermotropic liquid crystals. This field of work is beyond the scope of this contribution and deserves to be reviewed separately.

A classical example of surface freezing accompanied with incomplete wetting is the surface crystallization of long chain alkanes, which will be discussed in detail in section 3. There is an impressing amount of very accurate experimental data. Some computer simulations and thermodynamic theories are also available for these systems. However, a quantitative microscopic theory is still missing in this field. The opposite situation is encountered with colloidal solutions. There are very few systematic experimental studies on surface ordering effects, but there are microscopic theories available, which predict the surface phase behavior qualitatively correctly. These theories require the knowledge of the particle pair potential and the interaction potential of a particle with the surface. For colloidal particles the latter can in principle be determined experimentally by techniques such as atomic force microscopy (AFM) [33], total internal reflection microscopy (TIRM) [34] or the surface forces apparatus(SFA) [35]. Since in colloidal systems these interaction potentials can be of very simple nature, it might well be that experimental data on surface freezing of colloid solutions could provide the basis for theoretical investigations of the subject, which in turn could have a stimulating effect on the development of theories for low molar mass systems. In the light of this assumption this topical review is organized as follows.

After a brief outline of surface sensitive scattering techniques in section 2 , we will first focus on the surface ordering of simple chain molecules like long chain alkanes, alkenes and alcohols. Experimental findings, computer simulations and some theoretical approaches will be discussed in section 3. In section 4 mixtures and solutions of chain molecules will be treated. Finally we will review surface ordering effects in colloidal suspensions in section 5 .

\section{Scattering in reflection}

\subsection{General conventions}

Any surface disorder $\rightarrow$ order transition will yield a surface layer with a density which is different from the bulk value. Consequently, the electron density and the scattering length density for neutrons of the surface layer will also be different from the bulk. 


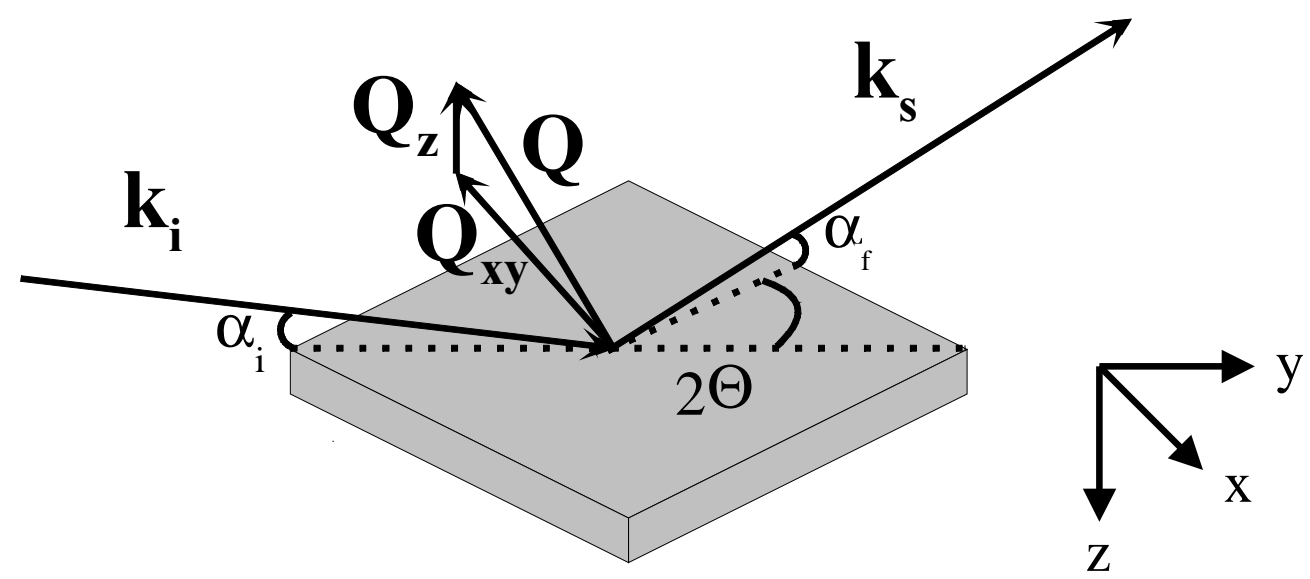

Figure 2. Definition of angles, real space directions and scattering vectors for a scattering experiment in reflection geometry.

Therefore surface sensitive scattering of x-rays or neutrons is the method of choice to study surface phase transitions which include a discontinuous change of an order parameter along the surface normal. In this section the principles of surface sensitive $\mathrm{x}-$ ray scattering will be briefly outlined with the understanding that the same formalism can be applied to neutrons as well.

The general scattering geometry for a scattering experiment in reflection is sketched in Figure 2. An incoming beam with the wave vector $\mathbf{k}_{i}$ impinges on the surface and the wave vector of the scattered beam $\mathbf{k}_{s}$ is determined by the position of the detection system which is fully defined by the in-plane angle $2 \theta$ and the reflection angle $\alpha_{f}$. The scattering vector is defined as $\mathbf{Q}=\mathbf{k}_{S}-\mathbf{k}_{i}$ which usually for convenience is split into an in-plane part and a part perpendicular to the surface $\mathbf{Q}=\mathbf{Q}_{x y}+\mathbf{Q}_{z}$. The norm of either part is then given by $\left|\mathbf{Q}_{x y}\right| \equiv Q_{x y}=4 \pi \sin \theta / \lambda$ and $\left|\mathbf{Q}_{z}\right| \equiv Q_{z}=4 \pi \sin \alpha_{z} / \lambda$ where $\lambda$ is the radiation wavelength. Since the refractive index for condensed matter is smaller than unity [36], the incident beam will be totally reflected if $\alpha_{i}<\alpha_{c}$, the critical angle of total external reflection. In this region the sample is penetrated only by an evanescent wave which propagates in the direction of the projection of the incident beam onto the surface. For $\alpha_{i} \ll \alpha_{c}$ the minimum $1 / e$-penetration depth is given by $\Lambda_{0}=\left(16 \pi r_{0} \rho_{e}\right)^{-1 / 2}$ where $r_{0}$ is the Thompson scattering length of a single electron and $\rho_{e}$ is the electron density of the sample. As shown in Figure 3 the penetration depth, $\Lambda$, increases when $\alpha_{i}$ approaches $\alpha_{c}$ and jumps several orders of magnitude on passing $\alpha_{c}$ until $\Lambda$ is only a function of the linear absorption coefficient of the sample at $\alpha_{i} \gg \alpha_{c}$. Thus it is possible to distinguish structural information either from a thin surface layer or from the sample bulk by simply varying the angle of incidence in a scattering experiment carried out in reflection geometry (XERD). Besides the XERD-experiment, where the full 3-D scattering is probed, there are two basic types of experiments which can be performed during which certain geometrical restrictions are applied, i. e. diffraction at grazing incidence (GIXD) and specular reflectivity (XR), which will described in the following sections. 
Surface or

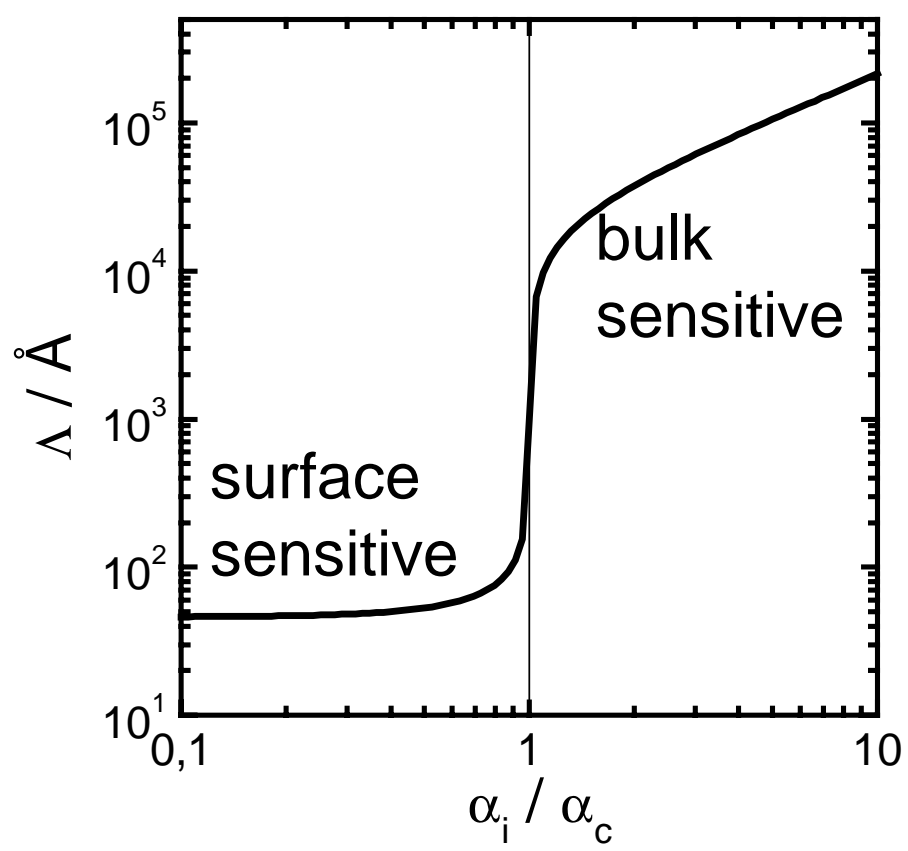

Figure 3. Penetration depth dependence on the angle of incidence of an x-ray beam with a wavelength of $\lambda=1.54 \AA\left(\mathrm{Cu}-\mathrm{K}_{\alpha}\right)$ impinging on a water surface. Due to the large variation of $\Lambda$ a scattering experiment carried out at $\alpha_{i} \ll \alpha_{c}$ is sensitive to structures close to the interface, while at $\alpha_{i} \gg \alpha_{c}$ it is sensitive to bulk structures.

\subsection{Grazing incidence diffraction}

GID was first developed to investigate surface reconstruction of solid surfaces $[37,38]$. It is a particularly powerful tool for the investigation of crystalline structures which are confined to a surface layer of well defined thickness, i. e. to two dimensions without a periodicity in the z-direction. In a scattering experiment on such a structure non-zero Bragg diffraction will occur if the incident wave propagates in the crystal plane and the $h, k$ lattice lines are oriented such that they make an angle $\theta_{h k}$ with the propagation direction of the incident beam with $\lambda=2 d_{h k} \sin \theta_{h k}$. Where $h$ and $k$ are the Millerindices of the two dimensional lattice and $d_{h k}$ is the corresponding lattice distance which is related to th e in-plane scatterin vector by $d_{h k}=2 \pi / Q_{x y}$. Experimentally this can be realized in GID where $\alpha_{i} \ll \alpha_{c}$ and the resulting evanescent wave is diffracted by the lateral structure of the surface layer.

In the hypothetical case that the thickness of the $2 \mathrm{D}$-crystal in the $\mathrm{z}$-direction is infinitely thin, all scattered waves which have the same $Q_{x y}$ will have the same phase in the Fraunhofer limit. Therefore diffracted intensity will not be limited to discrete spots like in the case of 3D-crystals, but will be evenly distributed along so called Bragg-rods, which are perpendicular to the surface and of infinite length. In real experiments however, the Bragg-rods will be of finite length due to the finite thickness of the crystalline layer.

For the examples to be discussed in this contribution, the two dimensional diffraction patterns can be analyzed to a good level of accuracy with the routines 
which have been developed in the field of GIXD-experiments from Langmiurmonolayers $[39,40]$. There the $2 \mathrm{D}$-crystals typically consist of a mono-molecular layer of chain molecules with a length of some tens of Aand rotational symmetry. Thus the molecules are regarded as cylindrical bodies which are arranged in a two dimensional array with hexagonal or centered rectangular unit cells in which the cylinder long axes have a finite tilt angle with respect to the surface normal. For untilted cylinders the particle scattering factor is a disc lying parallel to the surface. In this case the superposition of the Bragg-rods and the particle scattering factor leads to a maximum at $Q_{z}=0$ of the intensity distribution along the $z$-direction of the rod. If the molecules are tilted their particle scattering factor is tilted accordingly which leads to a maximum at $Q_{z}>0$. Thorough analysis of the of the Bragg-rods' intensity distribution along the $z$-direction eventually allows the determination of layer thickness, tilt direction and angle.

\subsection{Specular reflectivity}

In a specular reflectivity (XR) experiment the in-plane angle is kept at $2 \theta=0$ while the angle of incidence is varied typically from about zero to less than $3^{\circ}$. Below $\alpha_{c}$ the beam will be totally reflected, while for $\alpha_{i} \geq \alpha_{c}$ it will be partially refracted into the sample volume and partially reflected. The intensity reflected under specular conditions $\left(\alpha_{i}=\alpha_{z}\right), I_{\text {spec }}$, is recorded in dependence of the angle of incidence and normalized to the incident intensity, $I_{0}$, to yield the specular reflectivity as a function of $Q_{z}, I_{\text {spec }} / I_{0} \equiv R\left(Q_{z}\right)$. Since in this particular geometry only scattering vectors normal to the surface are probed, $R\left(Q_{z}\right)$ is the Fourier-transform of the electron density profile perpendicular to the surface $\rho_{e}(z)$.

$$
R\left(Q_{z}\right)=R_{F}\left(Q_{z}\right)=\left|\frac{1}{\rho_{e, \infty}} \int_{-\infty}^{\infty} \frac{d \rho_{e}(z)}{d z} \exp \left\{i Q_{z} z\right\} d z\right|^{2}
$$

Here $\rho_{e, \infty}$ is the electron density of the bulk material and $R_{F}\left(Q_{z}\right)$ is the Fresnelreflectivity, as defined by Equation (2) from a perfectly smooth surface of a sample with $\rho_{e, \infty}$.

In the case of ordered surface layers this profile can be interpreted in terms of the thickness and lateral mean electron density of the film. The effect of various electron density profiles $\rho_{e}(z)$ on the specular reflectivity is displayed in Figure 4. For a simple step-like profile, where the electron density jumps from zero to the bulk value $\rho_{e, \infty}$ the reflectivity decays monotonically following Fresnels' law

$$
R_{F}\left(Q_{z}\right)=\left|\frac{Q_{z}-\sqrt{Q_{z}^{2}-Q_{c}^{2}}}{Q_{z}-\sqrt{Q_{z}^{2}-Q_{c}^{2}}}\right|^{2}
$$

where $Q_{c}$ is the scattering vector corresponding to $\alpha_{c}$. A layer with enhanced electron density of thickness $d$ on top of the sample will cause undulations in the reflectivity curve. While the amplitude of these undulations is related to the difference $\rho_{e}-\rho_{e, \infty}$, the distance between two adjacent minima is roughly given by $\Delta Q_{z} \approx 2 \pi / d$. A parameterized description of the electron density profile can be obtained by nonlinear least squares fitting of a so called box- or slab-model. The formalism outlined above is usually referred to as the kinematic approximation. Although it neglects refraction and multiple scattering, it is accurate enough for most applications, if refraction is introduced a posteriori by using $Q_{z}=4 \pi \sin \alpha_{t} / \lambda$, where $\alpha_{t}$ is the angle of the refracted beam with the surface. For an exact formalism, the reader is referred 

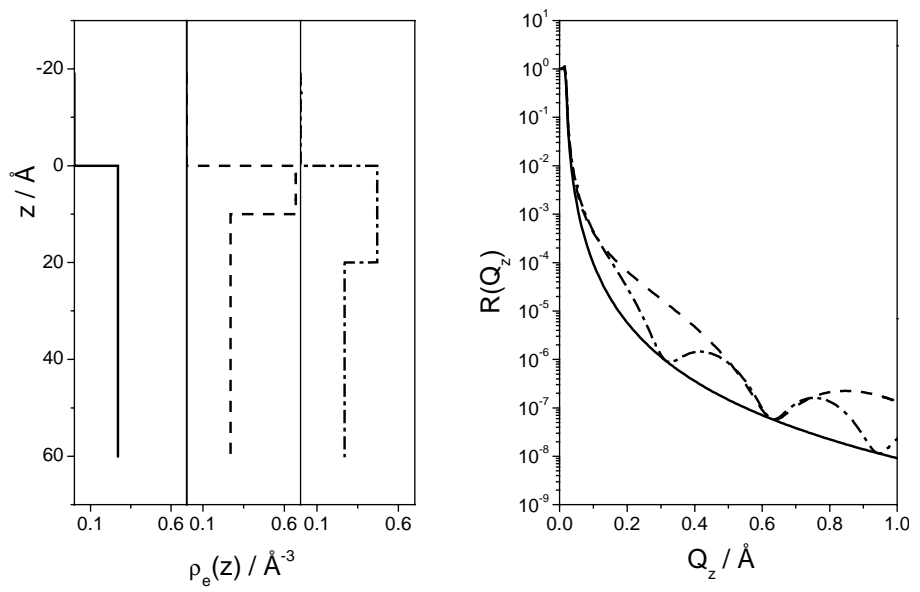

Figure 4. Left: Model profiles of the electron density. Right: Resulting reflectivity curves.

to the pioneering paper of Parratt [41] and for an overview of model independent approaches to data analysis see reference [42].

\section{Surface ordering of pure liquids}

\subsection{Alkanes}

Surface freezing of long chain alkanes was reported to manifest even in macroscopic observables. Shaking of melts of pure eicosane with a temperature up to $3 \mathrm{~K}$ above the melting temperature leads to foam formation. In the same temperature range macroscopic particles spread on the surface were seen to move with remarkable lateral speed (Marangoni flow). Performing quantitative measurements of the foam stability and the lateral speed as a function of temperature Gang et al [43] showed that both effects are related to the formation of a monomolecular quasi-crystalline layer at the liquid-vapor interface.

The first systematic study of surface freezing had been reported six years earlier by Earnshaw et al for melts of alkanes with carbon numbers $15 \leq n \leq 18$ [44]. They observed a positive temperature gradient of the surface tension, $d \gamma / d T$ directly above the bulk melting temperature of the alkane $T_{b}$, which became negative about three Kelvin above $T_{b}$. Since $d \gamma / d T$ is the negative surface excess entropy this finding was interpreted as surface induced pre-freezing which occurs at the temperature of the sign change, $T_{s}$. Further the authors argued that the surface freezing was limited to a few molecular length scales at most. The latter point was confirmed shortly later by Ocko et al, on the basis of XR-experiments [45,46]. Above $T_{s}$, the reflectivity curves show a monotonic decay, while they exhibit undulations in the temperature range $\Delta T=T_{s}-T_{b}$, as is shown in Figure 5 . The deduced electron density profiles show that there is a condensed layer on top of the sample with an electron density roughly 20 percent higher than the bulk value. The layer thickness corresponds nicely to the length of the hydrocarbon chains, indicating a monomolecular layer. In Figure 6 the reflected intensity at fixed $Q_{z}$ and the surface tension are plotted versus temperature. The surface freezing is clearly visible from the sudden increase of intensity at $T_{s}$ 


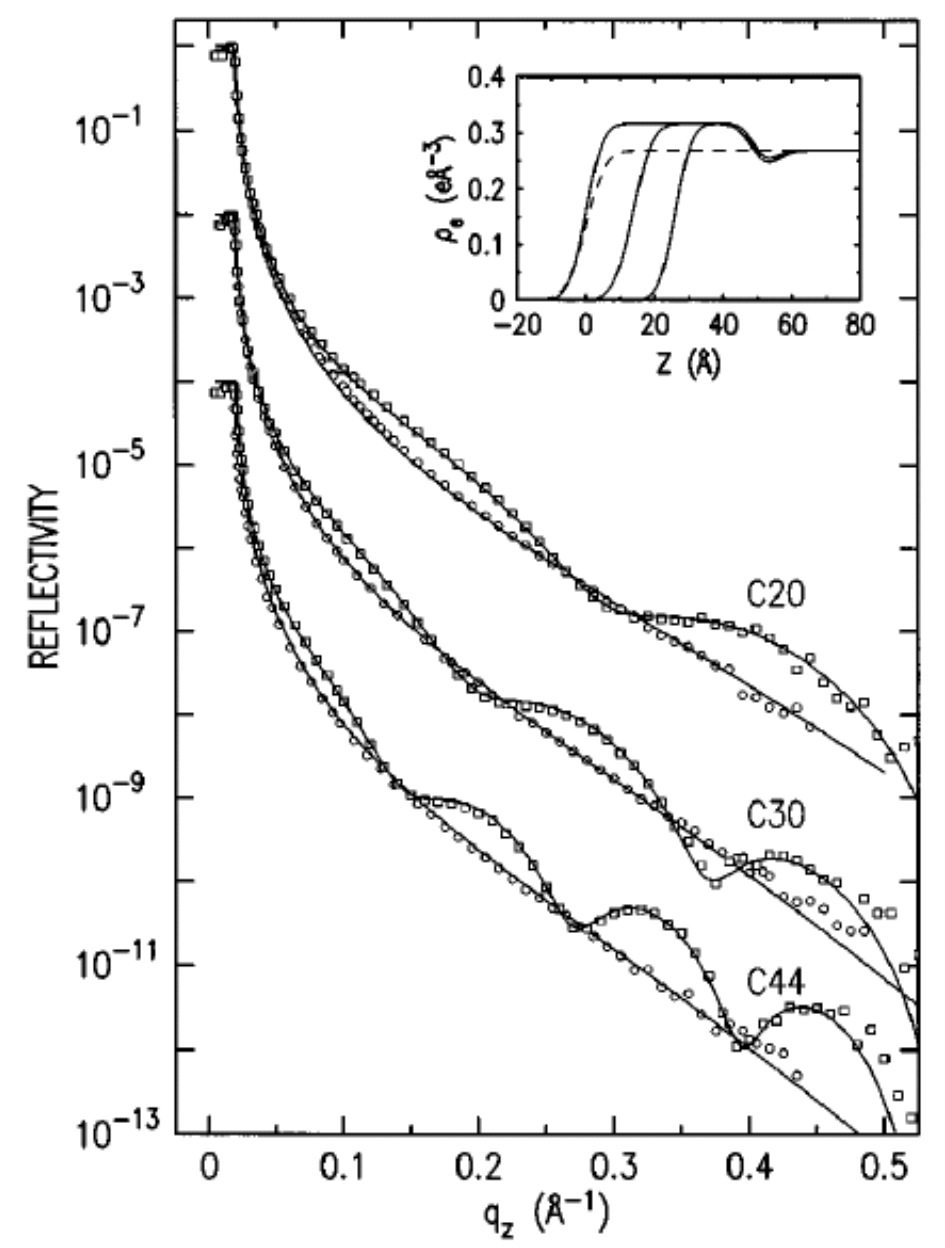

Figure 5. Reflectivity curves of akane surfaces with $n=20,30$ and 44 above $T_{s}$ (o) and at $T_{b}<T \leq T_{s}(\square)$. The lines are fits with a two-box model. The corresponding electron densities profiles shown in the inset are dashed above $T_{s}$ and solid for $T_{b}<T \leq T_{s}$ (Reproduced with permission from reference [48] (C) by the American Physical Society).

and the change of sign of the surface tension temperature gradient. Throughout the entire range of $\Delta T$ the reflectivity $d \gamma / d T$ remain constant indicating that no further structural changes occur.

Using GIXD the authors could further show that the surface layer was quasicrystalline with a positional correlation length in the micrometer range [45]. The complete series of hydrocarbons with carbon numbers covering a range of $14 \leq n \leq 50$ was subsequently studied by the same group [47,48]. Although there is some contradiction as to whether the smallest carbon number at which surface freezing occurs is $n=15$ [47] or $n=16$ [48], it is evident for the whole series that the surface frozen region is limited to a monomolecular layer for all carbon numbers investigated. That is the ordered phase does not wet the surface completely, contrary to what was observed for many liquid crystalline systems. 

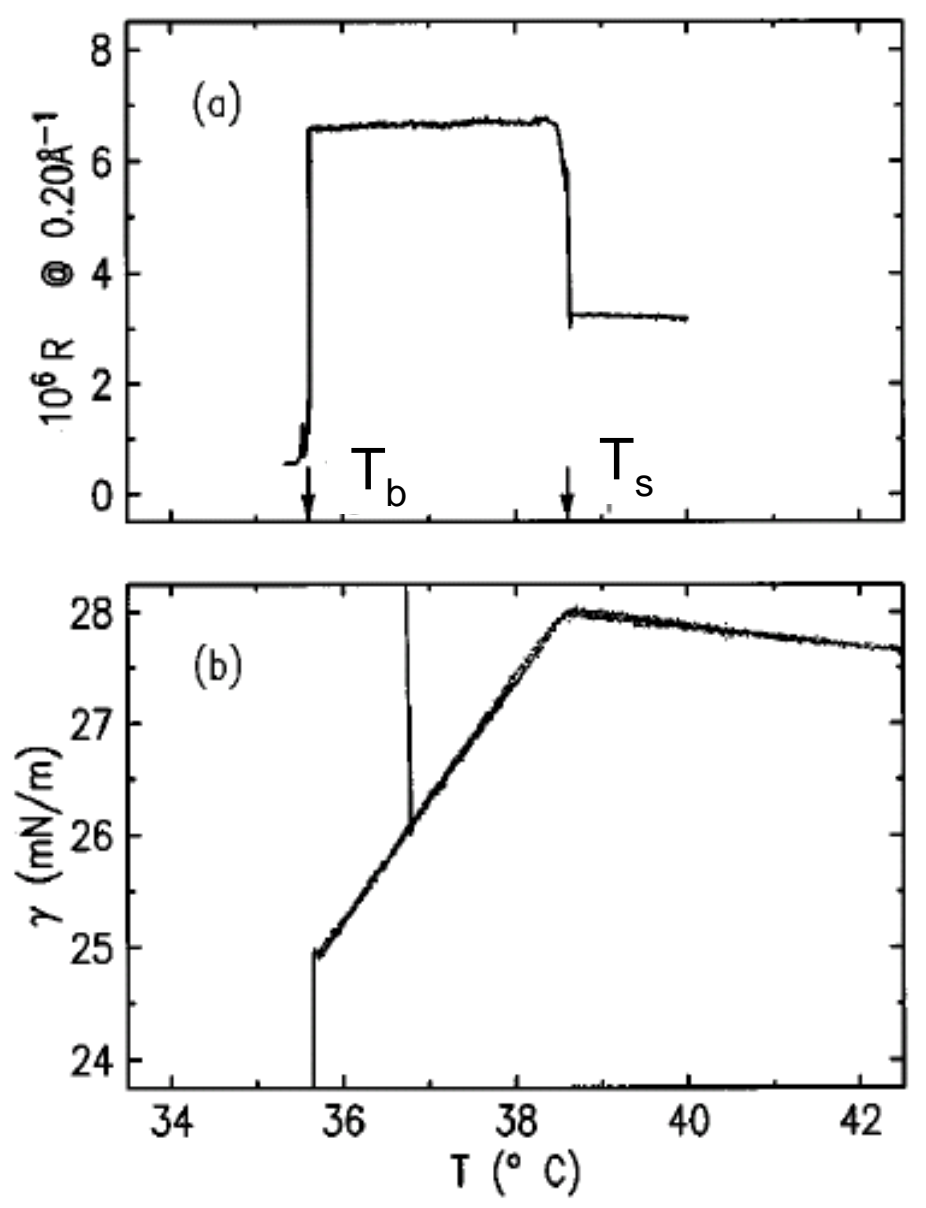

Figure 6. Temperature dependence of the reflectivity at fixed $Q_{z}$ (a) and the surface tension (b) of a $\mathrm{n}$-eicosane surface . The transition, from a liquid surface to a surface frozen layer at $T_{s}$, is manifest from the sharp intensity jump in (a) and the abrupt change of slope in (b). (Reproduced with permission from reference [48] (C) by the American Physical Society. Note that the transition temperature labels are exchanged in the original paper.)

The lateral structure of the monolayers depends on the carbon chain length. For $n<30$ it was reported to be a rotator phase of hexagonal symmetry with the molecules aligned parallel to the surface normal. In the range of $30 \leq n<44$ the structure is still a rotator phase with centered rectangular symmetry and the molecules are tilted towards their nearest neighbor. The tilt angle increases continuously with the chain length. Finally above $n=44$ the structure is crystalline with a next nearest neighbor tilt.

The temperature range, $\Delta T$, in which the surface layer exists increases steeply from zero at $n=15$ to roughly $3 \mathrm{~K}$ at $n \approx 20$, then decreases almost linearly to $\Delta T \approx 2.5 \mathrm{~K}$ at $n=44$ and finally drops of sharply to $\Delta T \approx 0.5 \mathrm{~K}$ at $n=50$. A semi-empirical model was derived which describes very well the dependence of $\Delta T$ on the carbon number for $n \leq 44$. The model is based on the variation of entropy and excess free energy in the surface layer with chain length, where the interaction energy 
is assumed to be of van der Waals type. The breakdown of this model is ascribed to the switch from a rotator like to a crystalline $2 \mathrm{D}$-structure of the layer at $n=44$.

On the other hand Pfohl et al came up with an alternative model for the surface frozen layer for heptadecane and eicosan [49]. They observed a change in the ellipsometric signal at $T_{s}$, which was however so small that the authors found it difficult to explain within the model discussed above. Therefore they suggested that the alkane chains in the surface near region are oriented perpendicular to the surface in a nematic like structure well above the surface freezing temperature. At $T_{s}$ a transition occurs from the nematic like to a smectic like monolayer with almost the same density as the nematic state. No penetration of the bulk phase into the monolayer is allowed which generates a density gap between the smectic surface layer and the underlying bulk. This gap causes x-ray interferences which can well describe the x-ray reflectivity experiments by $\mathrm{Wu}$ et al despite the equality of bulk- and surface density, which in turn allows for the observed small change of the ellipsometric signal at $T_{s}$.

Further studies applying surface forces measurements $[50,51]$ and differential thermal analysis and ultraviolet photoemission [52] also detected a surface phase transition accompanied by the stabilization of a more ordered phase towards higher temperature at the surface. However these techniques do not provide any information on the structure of the surface and thus these results can not help to distinguish the alternative structural models.

The situation is further complicated by the fact that the structure of the frozen surface layer appears to depend also on the nature of the spectator phase. Experiments on the interface between $\mathrm{SiO}_{2}$ covered silicon wafers and alkane melts suggested the formation of an interfacial layer in which the molecules are oriented perpendiular to the interface below $T_{s}[53,54]$. However, these findings were contradicted by Volkmann et al [56] in the sense that in the first layer on top of the $\mathrm{SiO}_{2}$ the molecules are oriented parallel to the interface, followed by a layer with perpendicular orientation. Parallel orientation of the first alkane layers is similar to what was reported earlier on normal alkanes adsorbed to solid substrates [57-60].

Using sum frequency generation spectroscopy Yeganeh [61] studied the phase transition and molecular arrangement of alkanes at an $\mathrm{Al}_{2} \mathrm{O}_{3}$ interface. It was observed that this particular interface does not change the transition temperatures from their bulk values. In the solid state the chain molecules lie on the substrate the $\mathrm{C}-\mathrm{C}$ axis parallel to the interface and the molecular plane perpendicular to the substrate normal.

The phenomenon of surface freezing has been tackled theoretically and by applying computer simulations by several groups. Ocko et al propose a simple model which explains surface freezing purely on the basis of the interfacial tensions of the two participating interfaces $[48,62]$, i. e. between the melt and the surface layer and between the surface layer and the vapor phase. On the other hand Tkachenko et al [63-65] propose that additional entropy contributions due to fluctuations of the alkane chains in the directions of their long axis have to be taken into account for the calculation of the free energy of the system.

Various simulation studies reproduced the experimental findings in part, but failed to in important details. All simulation studies find the formation of a frozen surface layer with perpendicular orientation of the chain molecules, however as opposed to the x-ray experiments Li et al find surface freezing for chains as short as $n=11[66,67]$. The simulation studies of Shimizu et al reveal that the surface layer is in thermal equilibrium with the melt through frequent molecular exchanges. Further it is shown that the molecules have high lateral mobility and a broad positional distribution of 
centers of mass along the surface normal. In this sense these simulations are in favor of the fluctuation scenario by Tkachenko et al. However, it was also found in this investigation, that slow stepwise cooling will lead to multi-layer formation, which is in clear contradiction to all experimental findings. Probably the best agreement so far with experiments was found in simulations of heptadecane by Smith et al . These authors also find that the properties of the surface chains are in accord with the model of a fluctuation stabilized layer [68]. The layer was found to be ordered, yet not crystalline and the molecules are able to diffuse in the plane of the surface and perpendicular to it. However, it is not clear whether the degree of order would allow for the resolution limited GID-peaks which have been observed experimentally [48].

\subsection{Alkenes, alcohols and semi-fluorinated alkanes}

In this section surface freezing of chain molecules, which are different from alkanes in either of two respects will be discussed. Alkanes interact mainly via van der Waals potentials and these interactions are invariant to the inversion of the molecules about their centers of mass. We will see in the following that breaking either of these conditions will grossly alter the structure and phase behavior of surface frozen layers.

The simplest case, where the symmetry of the chain is broken by the introduction of a $\mathrm{C}-\mathrm{C}$ double bond, was studied by Gang et al for the case of $\alpha$-eicosene [69]. They found that the structure of the surface frozen layer was very similar to that of the corresponding normal alkane. However the temperature range in which the surface layer is stable is larger for the $\alpha$-eicosene as compared to the saturated chain. This is ascribed to the entropy gain associated with flipping of the molecules, which is appearantly not over compensated by the corresponding energy cost.

A much greater variety of structures and phase behavior was observed for normal alcohols, i. e. hydrocarbon chains carrying an $\mathrm{OH}$-substituent at one of the terminal carbon atoms [70-72]. Surface freezing of alcohos was discovered first by Deutsch et al [70]. Also in the case of alcohols with even carbon numbers, a 2-D crystalline layer is formed which maintains constant thickness throughout the entire temperature range, $\Delta T$, in which it is stable. For chain lengths of $n \leq 22$ the lateral structure of the layer is hexagonal with the chains oriented perpendicular to the surface, while it is centered rectangular with a next-nearest neighbor tilt for longer chains.

With $\Delta T \lesssim 1 \mathrm{~K}$ it is somewhat smaller than observed for alkanes. However, the major difference to the behavior of alkanes concerns the structure along the surface normal. The surface frozen layer of an alcohol melt consists of two molecular sheets, i. e. it is a bilayer. The $\mathrm{x}$-ray reflectivity data show an enhanced electron density in the center of the layer corresponding to head-to-head stacking of the OH-groups. Analysis of the GID-data suggest that the bottom layer is displaced laterally with respect to the top layer, such that the $\mathrm{OH}$-groups of the bottom layer are located below the voids of the hexagonal unit cells of the top layer. This arrangement of the $\mathrm{OH}$-groups allows for the stabilization of the bilayer by hydrogen bonding. A second remarkable deviation from the alkane behavior is the fact that surface freezing occurs only with alcohols of even carbon numbers in the range $16 \leq n \leq 28$. This odd-even effect was ascribed to differences in the orientation of the terminal $\mathrm{OH}$-group relative to the molecular axis, which may render the formation of hydrogen bonds unfavorable in odd alcohols by making the relevant $\mathrm{HO}-\mathrm{H}$ distances deviate from the required value of $\sim 2.8 \AA[73]$.

As the capacity of hydrogen bond formation causes qualitative differences between 
the structure of surface frozen alkanes and alcohols, it is to be expected that additives which are able to form hydrogen bonds as well will further alter the surface structure of alcohol melts. Investigations of alcohols in equilibrium with a water saturated atmosphere $[71,72]$ show on one hand that the basic features of the bilayer structure are not changed except for a swelling of the bilayer due to intercalation of water into the $\mathrm{OH}$-group region. Normal to the surface the bilayers grow by $\sim 3 \%$ independently of the alcohol chain length. Parallel to the surface an expansion of the unit cell dimensions by $\sim 2 \%$ was observed only for the non-tilted systems. On the other hand an enormous increase of $\Delta T$ due to hydration was observed together with an expansion of the range of chain lengths in which surface freezing occurs. Different from the dry alcohols surface freezing occurs down to chain lengths of $n=12$ in hydrated systems and the temperature range is expanded roughly by a factor of two. These effects were accounted for quantitatively, by considering the hydration levels of the bulk and the surface, and taking into account the differences in that level due to adsorption or depletion of water at the surface. For a detailed description of this consideration the reader is referred to the paper by Gang et al [72]. The variability of phase behavior and structure of frozen surface layers may be further enhanced by suitable additives which will be discussed in section 4 .

A further class of chain molecules which show surface freezing are semifluorinated alkanes (SFA), i. e. carbon chains which are carrying hydrogen and fluorine atoms in a block wise arrangement; $\mathrm{F}_{m} \mathrm{H}_{n}$ where $m$ denotes the number of fluorinated carbon atoms and $n$ is the chain length of the hydrocarbon block. Like alkanes these molecules have only van der Waals interactions, but the symmetry of the interaction potential upon flipping the chains is severely perturbed. Miscibility studies of hydrocarbon and fluorocarbon solvents have shown [74] that the free energy of transfer of a $\mathrm{CH}_{2}-$ group into fluorocarbon and a $-\mathrm{CF}_{2}-$ group into hydrocarbon are approximately 1.1 and 1.4 $\mathrm{kJ}$ mol-1, respectively. These values imply that the antipathy between hydrocarbon and fluorocarbon chains is about $1 / 3$ of the free energy of transfer of $-\mathrm{CH}_{2}-$ groups from alkane to water. Further, the structure within the two blocks is different, a planar zig-zag chain in the $-\mathrm{CH}_{2}$-block with a cross sectional area of $\sim 18 \AA$ and a rigid helix in the $-\mathrm{CF}_{2}$-block with a lateral spatial requirement of $\sim 28 \AA$. A series of $\mathrm{F}_{12} \mathrm{H}_{n}$ (with $n=8,14$ and 19 ) melts was investigated with regard to surface freezing by Gang et al [75]. In the cases of $n=8$ and 14 the authors found surface frozen layers with a thickness which corresponds to the length of a completely stretched molecule, indicating a surface normal orientation. The electron density profiles deduced from XR-data by fitting to a two slab model clearly show that the F-blocks point to the vapor phase. However a detailed analysis of the profiles reveals that the two slabs cannot be simply identified with a close packing of the fluorinated and the hydrogynated block. Neither of the widths of the slabs coincide with the nominal lengths of the two blocks nor do the electron densities with those of closely packed Fblocks and H-blocks, respectively. These discrepancies were accounted for in a model of an up-down staggering of the molecules, which is probably brought about by the helical screw-like structure of the F-block. This would lead to lateral modulations of the surface frozen layer similar to that observed in the undulating lamellar phases of bulk $\mathrm{F}_{12} \mathrm{H}_{n}[76]$. The behavior of the long chained $\mathrm{F}_{12} \mathrm{H}_{19}$ was found to be even more complex. Contrary to all systems described so far the thickness and the density of the surface frozen layer vary in a complex manner with temperature. Surface tension measurements as well as XR-experiments indicate a continuous transition from the surface frozen to the liquid state. Further, no GID-spectra could be observed at any 


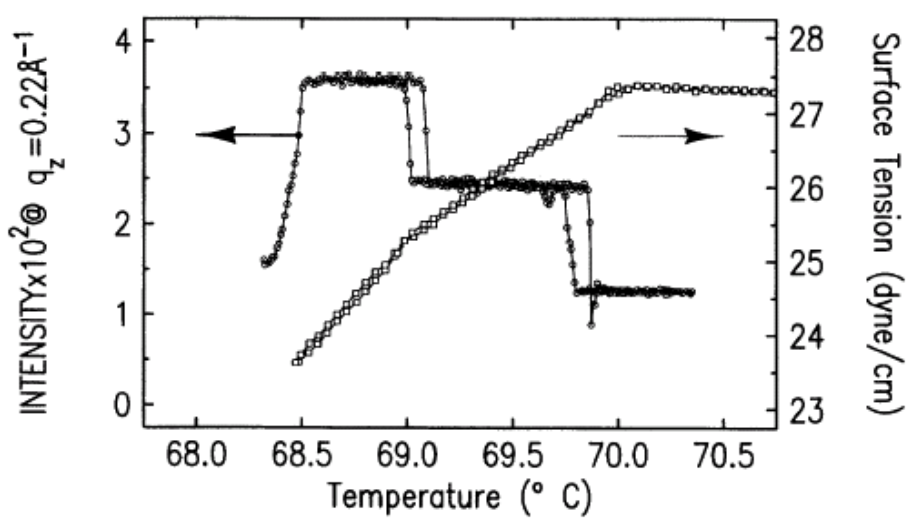

Figure 7. Temperature dependence of the reflectivity at a fixed $Q_{z}$ (left axis) and of the surface tension (right axis) from a $\mathrm{C}_{26}-\mathrm{C}_{36}-$ mixture at $\phi_{C_{26}}=0.41$. The formation of the surface frozen layer at $T_{s} \approx 70^{\circ} \mathrm{C}$ and the the transition to a new phase at $T_{n} \approx 69^{\circ} \mathrm{C}$ are seen as jumps in the reflected intensity and as slope changes in the surface tension. (Reproduced with permission from reference [77] (C) by the American Physical Society)

temperature indicating a disordered state of the surface layer. The authors suggest a buckled structure for this surface layer driven by the different cross sectional areas of the respective blocks and leading to a dome-covered surface with large voids. This model accounts for the temperature dependence of the layer thickness and the disordered structure as well as for the surprisingly low electron density which was observed.

\section{Liquid mixtures}

As we have seen in section 3 the structure and the phase behavior of surface frozen layers of chain molecules is dominated by a subtle energy-entropy balance. Consequently, one may expect novel effects in mixtures of chain molecules, since mixing introduces a further source of entropy.

Studies of mixtures of alkanes (denoted $\mathrm{C}_{n}$ and $\mathrm{C}_{n+\Delta n}$ ) with different chain lengths $n$ and $n+\Delta n$ by Wu et al $[77,78]$ show that the chain length mismatch, $\Delta n$, has drastic impact on the structure of the surface frozen layer. For small $\Delta n$ the properties of the surface layer change continuously from those of a pure $\mathrm{C}_{n+\Delta n}$-layer to those of a pure $\mathrm{C}_{n}$-layer with the bulk volume fraction of the short chain component, $\phi_{C_{n}}$. No further structural changes were observed either by varying temperature or as a function of bulk composition.

By contrast, mixtures with large $\Delta n$ show a discontinuous transition from a pure $\mathrm{C}_{n+\Delta n}$-layer to pure $\mathrm{C}_{n}$-layer as the bulk composition is varied and an additional new transition involving a change of tilt angle and direction which occurs as a function of bulk composition. As indicated in Figure 7 this transition can also be induced by temperature changes, which is a novelty for surface frozen layers of alkanes. The observed phenomena could be well accounted for in the framework of a Flory-Huggins type theory which is based on the balance of mixing entropy and repulsive energy due to the chain length mismatch.

A different scenario was observed for mixtures in which the short chain component 
does not show surface freezing in its pure state [79]. Here the surface frozen layer maintains the same structure as in the pure $\mathrm{C}_{n+\Delta n}$ case until it ceases to exist, if $\phi_{C_{n}}$ exceeds a certain value.

Mixtures of alcohols with different chain lengths show a qualitative similar $\Delta n$ dependence as alkanes $[80,81]$. However, there is a remarkable difference in that long chain alcohols induce surface freezing of alcohols which do not show surface freezing if they are pure. The addition of $10 \% \mathrm{C}_{18} \mathrm{OH}$ to $\mathrm{C}_{12} \mathrm{OH}$ induces the formation of a $\mathrm{C}_{12} \mathrm{OH}$ frozen bilayer in the appropriate temperature range [80]. In mixtures of $\mathrm{C}_{14} \mathrm{OH}$ and $\mathrm{C}_{18} \mathrm{OH}$ the properties of the surface frozen layer changes continuously from a pure $\mathrm{C}_{14} \mathrm{OH}$-bilayer to a pure $\mathrm{C}_{18} \mathrm{OH}$-bilayer with increasing bulk fraction of $\mathrm{C}_{18} \mathrm{OH}$ [81]. Note that neither pure $\mathrm{C}_{12} \mathrm{OH}$ nor pure $\mathrm{C}_{14} \mathrm{OH}$ show suface freezing, which indicates that the surface phase behavior of alcohol melts can be tuned by appropriate additives.

This effect was extensively studied by the addition of short chain $\alpha, \omega$-diols, i. e. hydrocabon chains carrying an $\mathrm{OH}$-group on both terminal carbons, to long chain alcohols [82-84]. It was observed that the surface frozen layers of alcohols can be effectively tuned from bilayer to mononolayer structure by addition of $\alpha, \omega$-diols with different chain lengths $m$. The resulting $(T, \phi, m)$ phase diagram is displayed in Figure 8. At molar fractions greater than 0.06 of $\alpha, \omega$-diol in $\mathrm{C}_{20} \mathrm{OH}-$ melts a monolayer phase appears when the samples are heated from the surface frozen state to the liquid state. The temperature range in which this monolayer exists becomes broader with increasing bulk concentration of the diol, while it shrinks with increasing carbon number $m$ of the diol. The observed phase diagram could be well predicted by a simple theory, which assumes a linearized dependence of the free energies of the various interfaces in each phase on the diol content.

The subtlety of the energy-entropy balance which determines the surface structure of alcohol melts is emphasized by the observation of a quadrilayer structure which was reported very recently [85]. Mixtures of $\mathrm{C}_{18} \mathrm{OH}$ and $\mathrm{C}_{26} \mathrm{OH}$ have a narrow range of compositions, $0.2 \lesssim \phi_{C_{2} 6} \lesssim 0.23$, in which cooling of the melt leads to the usual surface frozen bilayer which is followed by a quadrilayer structure before bulk freezing. It is not clear so far whether this is a hint of a chain length or composition driven wetting transition from partial to complete wetting.

A thermodynamic theory which accounts well for the surface phase behavior of a wide variety of alkane mixtures as well alcohol mixtures was given in two very recent papers $[86,87]$. The theory is based on a regular mixture approach for the bulk, which takes into account the entropy of mixing and a phenomenological energy contribution, $\omega$, due to the interchange of two alkane molecules with different chain lengths in the crystalline phase. To describe the surface phase behavior the composition of the surface (which is different from the bulk mixture) was calculated on the basis of the Gibbs adsorption rule. Balancing the chemical potentials of either component in either surface phase (equilibrium condition) yields expressions for the dependence of $T_{s}$ and the surface excess entropy, $\Delta S^{s}$ on the mixture composition with $\omega$ as the only adjustable parameter. Fitting of these expressions to the experimental data shows that $\omega$ depends linearly on $(\Delta n / \bar{n})^{2}$, where $\bar{n}$ is the mean chain length of the mixture. This behavior was found to be universal for all alkane mixtures, dry alcohol mixtures and even for hydrated alcohol mixtures, if the contribution of the water to the mixing entropy is taken into account.

For the sake of completeness we mention here that solutions of semi-fluorinated alkanes in hydrocarbons have also been investigated concerning their surface phase 


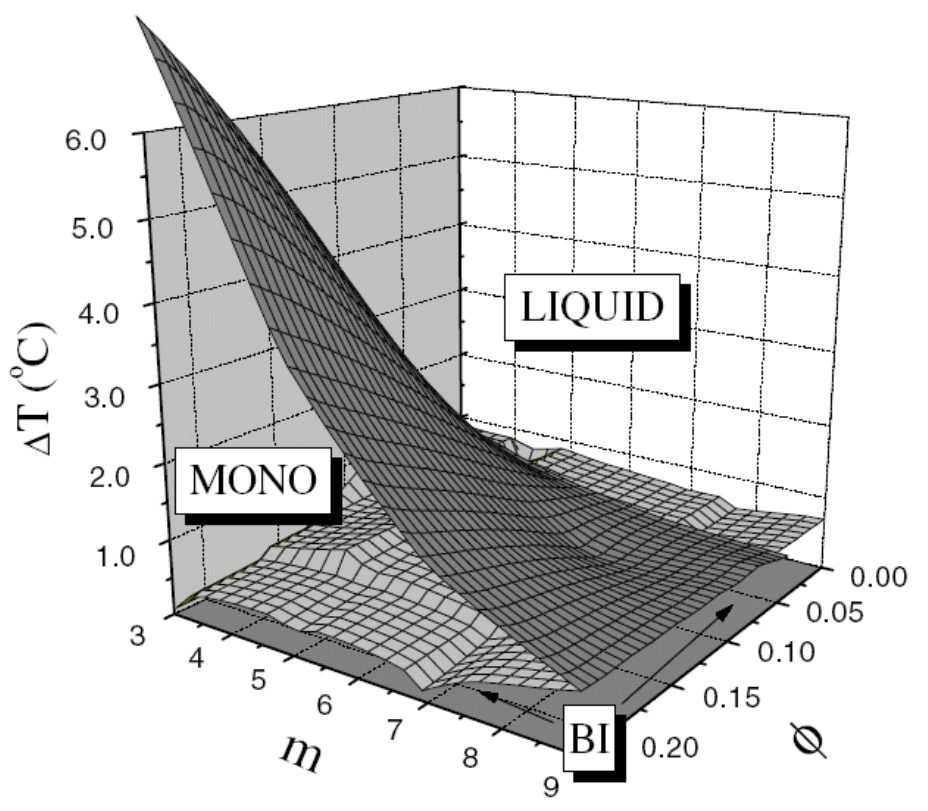

Figure 8. Surface phase diagram of $\alpha, \omega$-diol: $\mathrm{C}_{20} \mathrm{OH}-$ melts. $\Delta T$ is the temperature difference from the bulk freezing temperature $T_{f}, \phi$ is the molar fraction and $m$ is the chain length of the diol. (Reproduced with permission from [84]

behavior [88-91]. However, in these studies the formation of an ordered SFA surface layer was observed at very low concentrations. It is therefore very likely that the layer formation is adsorption driven and not a surface freezing effect.

\section{Colloidal suspensions}

Compared to the enormous number of studies of surface freezing effects in molecular liquids, there are only very few contributions on surface induced ordering of colloidal suspensions under comparable conditions. There are investigations on two dimensional colloidal systems,e. g. [92,93] and on surface induced ordering by patterned substrates, e. g. $[94,95]$. The present discussion will however be restricted to three dimensional dispersions which show surface ordering induced by an energetically homogeneous spectator phase.

Solutions of nonionic surfactants or amphiphilic blockcopolymers are particularly interesting for the investigation of surface effects, since they show a wealth of differently structured [96-99] lyotropic mesophases with relevant length scales in the colloidal domain. Surfactants of the oligo-(ethyleneoxide)-n-alkylether type with the general chemical composition $\mathrm{CH}_{3}\left(\mathrm{CH}_{2}\right)_{m-1}\left(\mathrm{OC}_{2} \mathrm{H}_{4}\right)_{n} \mathrm{OH}$ (abreviated $\mathrm{C}_{m} \mathrm{E}_{n}$ ) consist of a hydrocarbon tail which is hydrophobic and a water soluble oligo(ethylenoxide) headgroup. If dissolved in water they form micelles at concentrations above a so called critical micellization concentration $(\mathrm{cmc})$. Depending on the relative volumes of the molecular moieties the shape of these micelles can be either spherical, ellipsoidal or thread-like. Upon increasing concentration solutions containing thread-like micelles will transform into a lyotropic liquid crystalline phase, denoted $\mathrm{H}_{1}$, in which the 


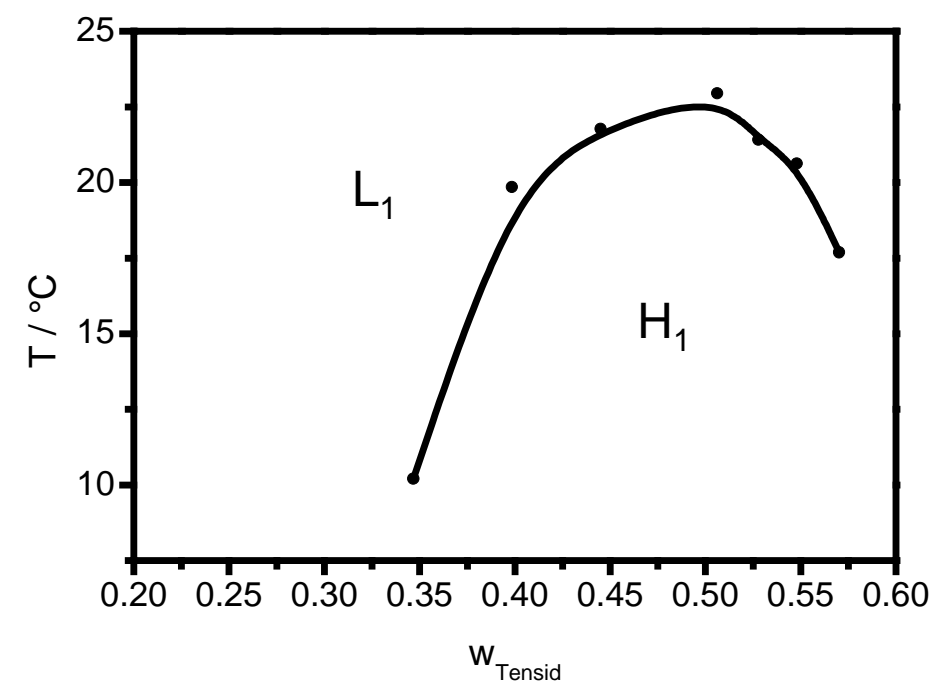

Figure 9. Coexistence line of the isotropic $\mathrm{L}_{1}$-phase with the hexagonal $\mathrm{H}_{1}$ phase in the bulk of aqueous $\mathrm{C}_{12} \mathrm{E}_{5}$ solutions. The data points were read off graphically from [100] and the full line is just a guide to the eye.

micelles are arranged on a two dimensional hexagonal array with their long axis aligned parallelly. The coexistence line of the isotropic micellar phase, $\mathrm{L}_{1}$, and $\mathrm{H}_{1}$-phase of the $\mathrm{C}_{12} \mathrm{E}_{5}+$ water is sketched in Figure 9 [100].

The effect of the macroscopic solution vapor interface on this coexistence line was investigated by Braun et al [101-104]. These investigations were to our knowledge the first systematic experimental studies of surface ordering effects in colloidal dispersions. The authors showed that the free surface has an impact on the phase behavior and the surface near structure in a threefold way.

First, the hexagonal arrays are forced to align such that the orientation of the (01) lattice plane is parallel to the surface with a variation of less than 3 degrees. This is demonstrated by the diffraction pattern shown in Figure 10 which was recorded in reflection geometry. The occurrence of Bragg diffraction peaks shows that there are only discrete orientations of lattice planes with respect to the surface plane. The surface alignment persists several micrometers away from the surface into the bulk. On the other hand, the cylinder long axes were shown to be oriented randomly in the surface plane. That is, the $\mathrm{H}_{1}$-phase has to be regarded as a powder sample in the surface plane and as being monocrystalline in the direction along the surface normal.

Second, closer inspection of Figure 10 reveals that the diffraction pattern consists of a set of hexagonally arranged doublet peaks, which is strong evidence for the existence of two hexagonal lattices with different lattice constants in real space. In Figure 11 the intensity of the (02) peak at various angles of incidence is plotted versus $Q_{z}$. On increasing the angle of incidence, i. e. going from surface sensitive to bulk sensitive scattering geometry, the peak at higher $Q_{z}$ decreases gradually. This shows that the lattice which causes this scattering peak is present only in a near surface region. Consequently, there has to be a layer close to the surface where the lattice constant is reduced as compared to the bulk, i. e. the surfactant volume fraction in this layer is higher than in the bulk. The authors speculate that the coexistence of two isostructural phases with different densities is due to the lateral pressure which 


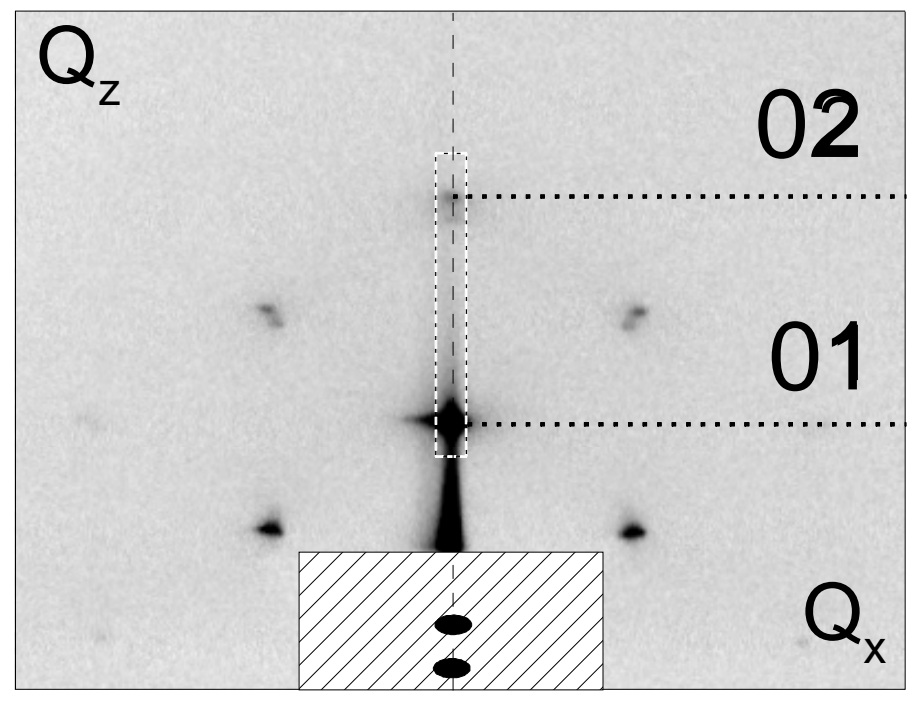

Figure 10. Two dimensional diffraction pattern recorded in reflection geometry from a $\mathrm{C}_{12} \mathrm{E}_{5}$ +water mixture with a surfactant content of $50 \%$ by weight at $22{ }^{\circ} \mathrm{C}$. Note the doublet character of all diffraction peaks. The numbers are the Miller indices of the peaks which have only a $Q_{z}$ contribution to the scattering vetor. The white rectangle indicates the location of the linear position sensitive detector which was used to record the spectra displayed in Figures 11 and 12 . The shaded area marks the position of a primary beam stop.

the micelles at the interface experience. A similar effect though in bulk dispersion has been reported by Bolhuis et al [105]. On the basis of Monte-Carlo simulations it was predicted that sterically stabilized colloids will undergo pressure driven isostructural phase transitions, if their pair interaction potential has a secondary potential minimum due to short range attractions. Accordingly, shrinking of the lattice constant as observed by Lang et al [102] might be induced by the pressure tensor asymmetry at the surface.

Third it was demonstrated that the system exhibits surface freezing. This is evident from Figure 12 where the intensity distributions of the (02) peak along the surface normal are displayed as a function of temperature. There is clearly a temperature range in which the diffraction peak at low $Q_{z}$ has vanished while the high$Q_{z}$ peak is still prominent. This shows clearly that the bulk solution is in the isotropic state while the surface region is in the hexgonal state at the same temperature.

From the second and third points it follows immediately that the $\mathrm{L}_{1}-$ $\mathrm{H}_{1}$ coexistence line is shifted to higher surfactant concentrations and to higher temperatures as compared to the bulk phase behavior.

As opposed to molecular melts, surface ordering in colloidal suspensions was observed also with spherical particles. This effect was first predicted on the basis of molecular dynamic simulations $[106,107]$ and experimentally reported by Gestenberg et al . These authors have have investigated solutions of spherical blockcopolymer micelles [108-110] which can be modeled as a system with hard body interacions. Deep in the isotropic region of the bulk phase diagram the authors observed a broad peak in the neutron reflectivity curve. Thorough data analysis revealed an undulating scattering length density profile along the surface normal which 


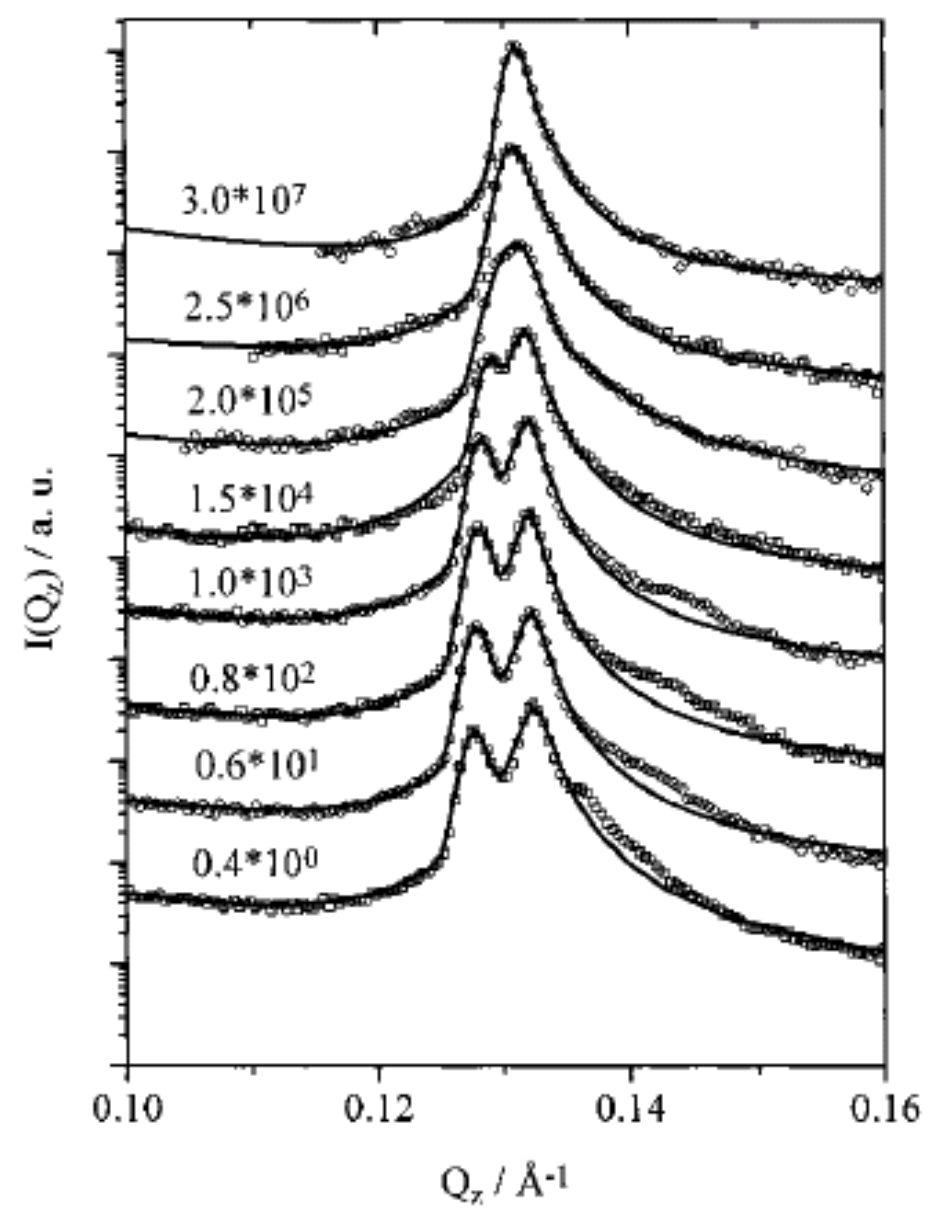

Figure 11. Intensity distribution of the (02) peak along the surface normal from a $\mathrm{C}_{12} \mathrm{E}_{5}+$ water mixture with a surfactant content of $50 \%$ by weight. A linear position sensitive detector was located at the position indicated by the white rectangle in Figure 10. The numbers above the curves indicate the multifolds of $\alpha_{c}$ at which the data were recorded multiplied with the factors used to shift the curves on the ordinate. The temperature was kept constant to $\pm 0.01 \mathrm{~K}$ (Reproduced with permission from reference [102] (c) by the American Chemical Society).

decays to the bulk value after about three to seven perids depending on the bulk composition. This is consistent with the formation of a layered structure of spherical micelles induced by the surface.

Similar observations were reported from aqueous dispersions of silica particles by Madsen et al [111]. Solutions with a colloid volume fraction of $\phi \approx 0.27$, i. e. roughly half the value required for bulk crystallization of a hard sphere suspension, were investigated applying XR and GID. The observed electron density profiles showed undulations with a period which coincides roughly with the particle diameter. On the other hand no diffraction peaks could be detected in GID. Therefore it is assumed that the particles arrange in layers which however do not have any lateral order.

Very recently Lang et al reported investigation on the isotropic to nematic 


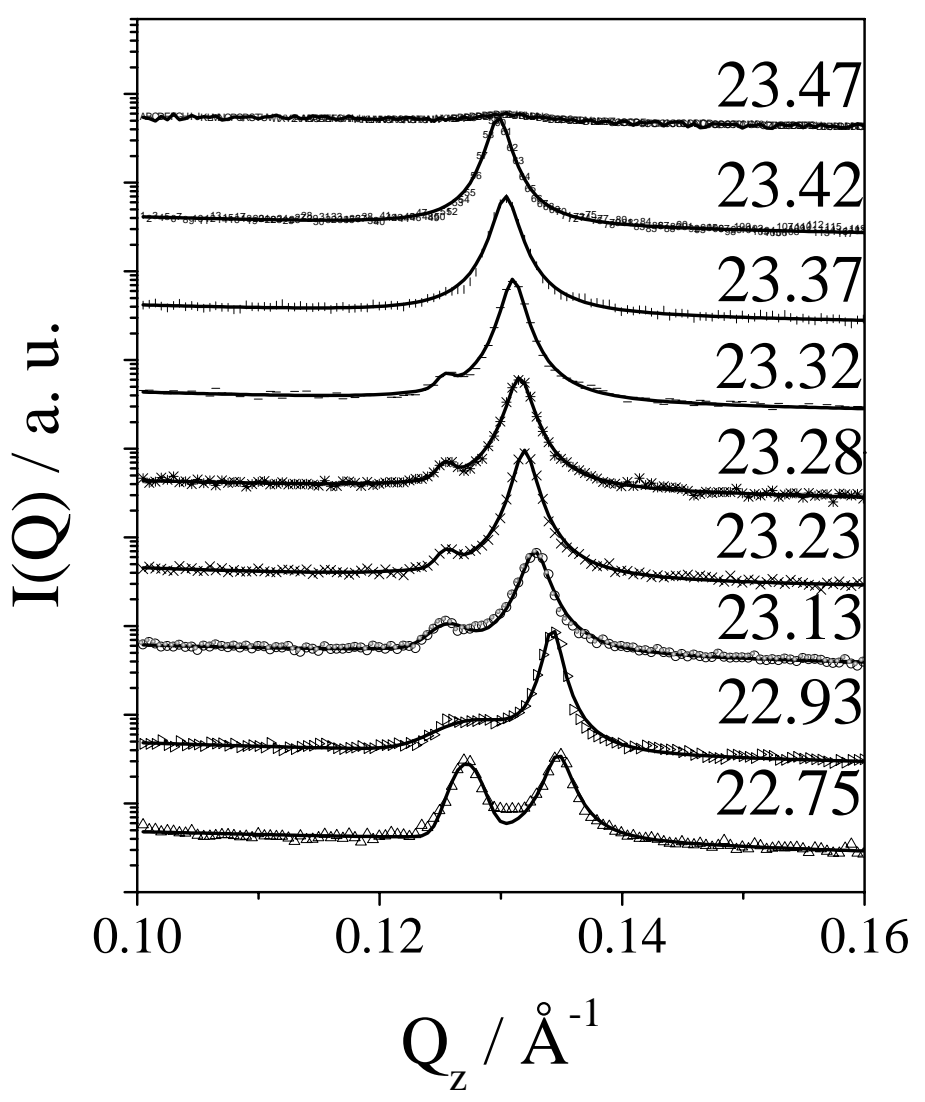

Figure 12. Intensity distribution of the (02) peak along the surface normal from a $\mathrm{C}_{12} \mathrm{E}_{5}+$ water mixture with a surfactant content of $50 \%$ by weight. The spectra were recorded at constant angle of incidence. The numbers indicate the sample temperature in ${ }^{\circ} \mathrm{C}$.

transition of thread-like blockcopolymer micelles close to a solid wall [112]. They find the formation of a nematic layer adjacent to the wall at a volume fraction which is roughly $85 \%$ of the volume fraction required to induce the nematic phase in the bulk. This is in qualitative agreement with theoretical prediction and simulations for hard rod suspensions by van Roij et al and Dijkstra et al [113,114]. However, the thickness of the observed nematic layer was not in the range of the thread contour length, as to be expected from the theoretical prediction, but rather in the range of the threads persistence length. The latter finding is in good agreement with the theoretical predictions by Chen et al which take into account the finite flexibility of the micelles $[115,116]$

\section{Conclusions}

To summarize, we have collected experimental reports and some theoretical as well as computer simulation approaches on surface ordering phenomena in soft condensed matter systems. The broadest experimental basis clearly exists for melts of linear chain molecules. The variety of surface phase behavior and structural properties increases 
rapidly with the complexity of molecular interactions and shape. A quantitative microscopic theory based on experimental knowledge of the molecular interaction is still not available. On the other hand this kind of theory is available for colloidal suspensions, as in these cases the pair interaction potentials are often sufficiently simple, to allow for a thorough microscopic description. Further, surface ordering effects in colloidal suspensions have gained increasing interest from experimentalists over the last decade and we are therefore confident that further experimental investigations in this field will promote our understanding of these effects in general.

\section{Acknowledgments}

My contribution to the field of surface induced ordering effects did greatly benefit from the work of the former PhD-students Chr Braun and P Marczuk, whom I thank for the collaboration. Many stimulating discussions with numerous colleagues in particular with R Steitz, H Rhan and my former mentor G H Findenegg are thankfully acknowledged. Last but not least I thank $\mathrm{H}$ Löwen for the invitation to write this topical review.

\section{References}

[1] Ninham B W 1999 Adv. Colloid Interface Sci. 831

[2] Penfold J 2001 Rep. Prog. Phys. 64777

[3] Frenken J W M and van der Veen F F, 1985 Phys. Rev. Lett. 54134

[4] Regan M J, Kawamoto E H, Lee S, Pershan P S, Maskil N, Deutsch M, Magnussen O M, Ocko B M and Berman L E 1995 Phys. Rev. Lett. 752498

[5] Regan M J, Pershan P S, Magnussen O M, Ocko B M, Deutsch M and Berman L E 1996 Phys. Rev. B $\mathbf{5 4 9 7 3 0}$

[6] Regan M J, Tostmann H, Pershan P S, Magnussen O M, DiMasi E, Ocko B M and Deutsch M 1997 Phys. Rev. B 5510786

[7] Ercolessi F, Di Tolla F D and Tosatti E 1997 Surf. Rev. Lett. 4833

[8] Celestini F, Ercolessi F and Tosatti E 1997 Surf. Sci. 377914

[9] Tostmann H, DiMasi E, Pershan P S, Ocko B M Shpyrko O G and Deutsch M 1997 Phys. Rev. B $\mathbf{5 9} 783$

[10] Turchanin A, Freyland W and Nattland D 2002 Phys. Chem. Chem. Phys. 4647

[11] Kroes G J 1992 Surf. Sci. 275365

[12] Elbaum M, Lipson S G and Dash J G 1993 J. Cryst. Growth 129491

[13] Lied A, Dosch H, Bilgram J H 1994 Phys. Rev. Lett. 723554

[14] Lied A, Dosch H, Bilgram J H 1994 Physica B 19892

[15] Dosch H, Lied A, Bilgram J H 1995 Surf. Sci. 327145

[16] Dosch H, Lied A, Bilgram J H 1996 Surf. Sci. 36643

[17] Furukawa J and Nada H 1997 J. Phys. Chem. B 1016167

[18] Makkonen L 1997 J. Phys. Chem. B 1016196

[19] Wilson P W, Arthur J W and Haymet A D J 1999 Biophys. J. 772850

[20] Maruyama M, Sato T, Taniguchi S, Kawamura M, Kodera S, Kishimoto Y and Furukawa Y 2000 Japan. J. Appl. Phys. 396696

[21] Doppelschmidt A and Butt H J 2000 Langmuir 166709

[22] Wei X, Miranda P B and Shen Y R 2001 Phys. Rev. Lett. 561554

[23] Wei X, Miranda P B, Zhang C and Shen Y R 2002 Phys. Rev. B 66085401

[24] Sadtchenko V and Ewing G E 2003 Can. J. Phys. 81333

[25] Suzanne J, Ferry D, Demirdjan B, Girardet C, Toubin C, Picaud S and Hoang P N M 2003 Can. J. Phys. $\mathbf{8 1} 415$

[26] Miyano K 1979 Phys. Rev. Lett. 4351

[27] Miyano K 1979 J. Chem. Phys. $\mathbf{7 1} 4108$

[28] Als-Nielsen J, Christensen F and Pershan P S 1982 Phys. Rev. Lett. 481107

[29] Pershan P S and Als-Nielsen J 1984 Phys. Rev. Lett. 52759

[30] Lucht R and Bahr Ch 1997 Phys. Rev. Lett. 783487 
[31] Lucht R, Bahr Ch, Heppke G and Goodby J W 1998 J. Chem. Phys. 1083716

[32] Schadt M and Helfrich W 1970 Appl. Phys. Lett. 18127

[33] Kappl M and Butt H J 2002 Part. Part. Syst. Char. 19129

[34] Prieve D C 1999 Adv. Colloid Interf. Sci. 8293

[35] Claesson P M, Ederth T, Bergeron V and Rutland M W 1996 Adv. Colloid Interf. Sci. 67119

[36] Warren B E 1990 X-ray Diffraction (New York: Dover Publications)

[37] Marra W C, Eisenberger P and Cho A Y 1979 J. Appl. Phys. 506927

[38] Eisenberger P and Marra W C 1981 Phys. Rev. Lett. 461081

[39] For a brief introduction see Kjær C 1994 Physica B $\mathbf{1 9 8} 100$

[40] For an overview see Als-Nielsen J, Kjær C, Leveiller F, Lahav M and Leiserowitz L 1994 Phisics Reports 246251

[41] Parratt L G 1954 Phys. Rev. 95359

[42] Lang P 1999 Amphiphiles at Interfaces Studied by Surface Sensitive X-Ray Scattering in Modern Characterization Methods of Surfactant Systems; Surfactant Science Series vol 83 ed Binks B P (New York: Marcel Dekker) pp 375-413

[43] Gang H, Patel J, Wu X Z, Deutsch M, Gang O, Ocko B M and Sirota E B 1998 Europhys. Lett. 43314

[44] Earnshaw J C and C. J. Hughes C J 1992 Phys. Rev. A 464494

[45] Wu X Z, Sirota E B, Sinha S K, Ocko B M and Deutsch M 1993 Phys. Rev. Lett. 70958

[46] Wu X Z, Ocko B M, Sirota E B, Sinha S K, Deutsch M, Cao B H and Kim M W 1993 Science 2611018

[47] Wu X Z, Ocko B M, Sirota E B, Sinha S K and Deutsch M 1993 Physica A 200751

[48] Ocko B M, Wu X Z, Sirota E B, Sinha S K, Gang O and Deutsch M 1997 Phys. Rev. E 55 3164

[49] Pfohl T, Beaglehole D and Riegler H 1996 Chem. Phys. Lett. 26082

[50] Maeda N, Kohonen M H and Christenson H K 1999 Phys. Rev. E 617239

[51] Maeda N, Kohonen M H and Christenson H K 2001 J. Phys. Chem B 1055906

[52] Yamamoto Y, Ohara H, Kajikawa K, Ishii H, Ueno N, Seki K and Ouch Y 1999 J. Electr. Spectr. Rel. Phen. 101555

[53] Merkl C, Pfohl T, Riegler H 1997 Phys. Rev. Lett. 794625

[54] Holzwarth A, Leporatti S and Riegler H 2000 Europhys. Lett. 52653

[55] Schollmeyer H, Struth B and Riegler H 2003 Langmuir 195042

[56] Volkmann U G, Pino M, Altamirano L A, Taub H and Hansen F Y 2002 J. Chem. Phys. 116 2107

[57] Kern H, v. Rybinski W and Findenegg G H 1977 J. Colloid Interface Sci. 59301

[58] Grosse-Rhode M and Findenegg G H 1978 J. Colloid Interface Sci. 64374

[59] Weckesser J, Fuhrmann D, Weiss K, Wöll C and Richardson N V 1997 Surf. Rev. Lett. 4209

[60] Wu Z, Ehrlich S N, Matthies B, Herwig K w, Pengcheng Dai, Volkmann U G, Hansen F Y and Taub H 2001 Chem. Phys. Lett. 348168

[61] Yeganeh M S 2002 Phys. Rev. E 66041607

[62] Sirota E B, Wu X Z, Ocko B M and Deutsch M 1997 Phys. Rev. Lett. 79531

[63] Tkachenko A V and Rabin Y 1996 Phys. Rev. Lett. 762527

[64] Tkachenko A V and Rabin Y 1997 Phys. Rev. E 55778

[65] Tkachenko A V and Rabin Y 1997 Phys. Rev. Lett. 79532

[66] Li H Z and Yamamoto T 2001 J. Chem. Phys. 1145774

[67] Li H Z and Yamamoto T 2002 J. Phys. Soc. Japan 711083

[68] Smith P, Linden-Bell R M, Earnshaw J C and Smith W 1999 Mol. Phys. 96249

[69] Gang H,Gang O, Shao H H, Wu X Z, Patel J, Hsu C S, Deutsch M, Ocko B M and Sirota E B 1998 J. Phys. Chem. B 1022754

[70] Deutsch M, Wu X Z, Sirota E B, Sinha S H, Ocko B M and Magnussen O M 1995 Europhys. Lett. 30283

[71] Gang O, Ocko B M, Wu X Z, Sirota E B and Deutsch M 1998 Phys. Rev. Lett. 801264

[72] Gang O, Wu X Z, Ocko B M, Sirota E B and Deutsch M 1998 Phys. Rev. E 586086

[73] Wang J L, Leveiller F, Jacquemain D, Kjær, Als-Nielsen J, Lahav M and Leiserowitz L 1994 J. Am. Chem. Soc. 1161192

[74] Binks B P, Fletcher P D I, Kotsev S N and Thompson R L 1997 Langmuir 136669

[75] Gang O, Ellmann J, Möller M, Kraack H, Sirota, E B, Ocko B M and Deutsch M 2000 Europhys. Lett. 49761

[76] Marczuk P and Lang P 1998 Macromolecules 319013

[77] Wu X Z, Ocko B M, Tang H, Sirota E B, Sinha S K and Deutsch M 1995 Phys. Rev. Lett. 75 1332 
[78] Wu X Z, Ocko B M, Deutsch M, Sirota E B and Sinha S K 1996 Physica B 221261

[79] Sloutskin E, Sirota E B, Kraack H, Ocko B M and Deutsch M 2001 Phys. Rev. E 64031708

[80] Doerr A, Wu X Z, Ocko B M, Sirota E B, Gang O and Deutsch M 1997 Colloids and Surfaces A : Physicochem. Eng. Aspects $\mathbf{1 2 8} 63$

[81] Sloutskin E, Sirota E B, Kraack H, Gang O, Doerr A, Ocko B M and Deutsch M 2002 J. Chem. Phys. 1168056

[82] Gang O, Ocko B M, Wu X Z, Sirota E B and Deutsch M 99 Phys. Rev. Lett. 82588

[83] Gang O, Ocko B M, Wu X Z, Sirota E B and Deutsch M 2000 Colloids and Surfaces A: Physicochem. Eng. Aspects $\mathbf{1 6 4} 55$

[84] Gang O, Ocko B M, Wu X Z, Sirota E B and Deutsch M 2000 J. Phys.: Condens. Matter 12 A357

[85] Sloutskin E, Kraack H, Gang O, Ocko B M, Sirota E B and Deutsch M 2003 J. Chem. Phys. 11810729

[86] Sloutskin E, Wu X Z, Peterson T B, Gang O, Ocko B M, Sirota E B and Deutsch M 2003 Phys. Rev. E 68031605

[87] Sloutskin E, Gang O, Kraack H, Doerr, A, Sirota E B, Ocko B M and Deutsch M 2003 Phys. Rev. E $\mathbf{6 8} 031606$

[88] Binks B P, Fletcher P D I, Sager W F C and Thompson R L 1995 Langmuir 11977

[89] Binks B P, Fletcher P D I and Thompson R L 1996 Ber. Bunsen- Ges. Phys. Chem. 100232

[90] Hayami Y and Findenegg G H 1997 Langmuir 134865

[91] Marczuk P, Lang P, Findenegg G H, Mehta S K and Möller M 2002 Langmuir 186830

[92] Pieranski P 1980 Phys. Rev. Lett. 45569

[93] Zahn K, Lenke R and Maret G 1999 Phys. Rev. Lett. 822721

[94] van Blaaderen A, Ruel R and Wiltzius P 1997 Nature 385321

[95] Heni M and Löwen H 2001 J. Phys.: Condens. Matter 134675

[96] Mitchell D J, Tiddy G J T, Waring L, Bostock T and MacDonald M P 1983 J. Chem. Soc. Farad. Trans. I $\mathbf{7 9} 975$

[97] Sjöblom J, Stenius P and Danielsson I 1997 Phase Equilibria of Nonionic Surfactants and the Formation of Microemulsions in Nonionic Surfactants Physical Chemistry; Surfactant Science Series vol 23 ed Schick M (New York: Marcel Dekker) pp 369-434

[98] Won, Y. Y.; Davis, H. T.; Bates, F. S. Science 1999, 283, 960.

[99] Alexandridis P and Lindman B. 2000 Amphiphilic Block Copolymers: Self-Assembly and Application (New York: Elsevier)

[100] Strey R, Schomäcker R, Roux D, Nallet F and Olsson U 1990 J. Chem. Soc. Farad. Trans. I 862253

[101] Braun C, Lang P and Findenegg G H 1995 Langmuir 11764

[102] Lang P, Braun Chr, Steitz R, Findenegg G H and Rhan H 1998 J. Phys. Chem. B 1027590

[103] Lang P 1999 J. Phys. Chem. B 1035100

[104] Lang P, Steitz R and Braun Chr 2000 Colloids and Surfaces A: Physicochemical and Engineering Aspects $\mathbf{1 6 3} 91$

[105] Bolhuis P, Hagen M and Frenkel D 1994 Phys. Rev. E 504880

[106] Courtemanche D J and van Swol F 1992 Phys. Rev. Lett. 692078

[107] Courtemanche D J, Pasmore T A and van Swol F 1993 Mol. Phys. 80861

[108] Gerstenberg M C, Pedersen J S and Smith G S 1998 Phys. Rev. E 588028

[109] Gerstenberg M C and Pedersen J S 2001 Langmuir 177040

[110] Gerstenberg M C, Pedersen J S, Majewski J and Smith G S 2002 Langmuir 184933

[111] Madsen A, Konovalov O, Robert A and Grübel 2001 Phys. Rev. E 64061406

[112] Lang P, Willner L, Pyckhout-Hintzen W and Krastev R 2003 Langmuir 197597

[113] van Roij R, Dijkstra M and Evans R 2000 J. Chem. Phys. 1137689

[114] Dijkstra M, van Roij R and Evans R 2001 Phys. Rev. E 631703

[115] Cui S M, Akcakir O and Chen Z Y 1995 Phys. Rev. E 514548

[116] Chen Z Y and Cui S M 1995 Phys. Rev. E 523876 